Check for updates

Cite this: RSC Adv., 2019, 9, 2919

Received 6th December 2018

Accepted 15th January 2019

DOI: $10.1039 / \mathrm{c} 8 \mathrm{ra10029b}$

rsc.li/rsc-advances

\section{Biochemical characterization and biocatalytic application of a novel D-tagatose 3-epimerase from Sinorhizobium sp. $\uparrow$}

\author{
Zhangliang Zhu, ${ }^{a}$ Chao Li, ${ }^{a}$ Xin Liu, ${ }^{a}$ Dengke Gao, ${ }^{a}$ Xueyu Wang, ${ }^{a}$ Masaru Tanokura, ${ }^{\text {ab }}$ \\ Hui-Min Qin (DD *a and Fuping Lu*a
}

Sinorhizobium SP. D-tagatose 3-epimerase (SDTE) catalyzes the conversion of D-tagatose to D-sorbose. It also recognizes $\mathrm{D}$-fructose as a substrate for $\mathrm{D}$-allulose production. The optimal temperature and $\mathrm{pH}$ of the purified sDTE was $50{ }^{\circ} \mathrm{C}$ and 8.0, respectively. Based on the sDTE homologous model, Glu154, Asp187, Gln213, and Glu248, form a hydrogen bond network with the active-site $\mathrm{Mn}^{2+}$ and constitute the catalytic tetrad. The amino acid residues around $\mathrm{O}-1,-2$, and -3 atoms of the substrates ( $\mathrm{D}$-tagatose/ $\mathrm{D}$-fructose) are strictly conserved and thus likely regulate the catalytic reaction. However, the residues at O-4, -5 , and -6 , being responsible for the substrate-binding, are different. In particular, Arg65 and Met9 were found to form a unique interaction with O-4 of D-fructose and D-tagatose. The whole cells with recombinant SDTE showed a higher bioconversion rate of $42.5 \%$ in a fed-batch bioconversion using $D$ fructose as a substrate, corresponding to a production of $476 \mathrm{~g} \mathrm{~L}^{-1} \mathrm{D}$-allulose. These results suggest that SDTE is a potential industrial biocatalyst for the production of $\mathrm{D}$-allulose in fed-batch mode.

\section{Background}

Rare sugars (i.e. xylitol, erythritol, L-ribose, D-tagatose, and $\mathrm{D}^{-}$ allulose) are defined as monosaccharides and their derivatives are infrequently found in nature. They have great potential use in the food and pharmaceutical industries. For instance, Dallulose (D-psicose or D-ribo-2-hexulose) is considered an ideal substitute for sucrose as an ultra-low calorie sweetener. ${ }^{1}$ It is absorbed in the small intestine and excreted from the body via the urine..$^{2-4}$ D-Allulose reduces the oxidation occurring via Maillard reaction during food processing, and thus increases the pleasantness of the food flavor. ${ }^{5} \mathrm{D}$-Tagatose can be used as a low-calorie sweetener in non-chronically administered drugs, mouth wash, and tooth paste, as well as a wide variety of health foods and dietary supplements. ${ }^{6}$ Furthermore, it is also a potential new antidiabetic and obesity control drug. ${ }^{7}$ Compared with the chemical synthesis of rare sugars, which

\footnotetext{
${ }^{a}$ Key Laboratory of Industrial Fermentation Microbiology of the Ministry of Education, Tianjin Key Laboratory of Industrial Microbiology, College of Biotechnology, Tianjin University of Science and Technology, National Engineering Laboratory for Industrial Enzymes, Tianjin 300457, P. R. China. E-mail: huiminqin@tust.edu.cn; lfp@tust.edu.cn; Fax: +86-22-60602298; Tel: +86-22-60601958

${ }^{b}$ Laboratory of Basic Science on Healthy Longevity, Department of Applied Biological Chemistry, Graduate School of Agricultural and Life Sciences, The University of Tokyo, 1-1-1 Yayoi, Bunkyo, Tokyo 113-8657, Japan. E-mail: amtanok@mail.ecc. u-tokyo.ac.jp

$\dagger$ Electronic supplementary information (ESI) available: The data collected upon which this article is based upon are all included in this manuscript and the additional files associated with it. See DOI: 10.1039/c8ra10029b
}

generates chemical waste and by-products, as well as requires complex multi-step reactions and the protection-deprotection of functional groups, ${ }^{8}$ the biosynthesis of rare sugars by enzymatic pathways is more environmentally friendly, since it can be done under moderate reaction conditions, offering high specificity, and sustainability.

In 2002, the creative izumoring strategy was established, through which all rare sugars and their derivatives could be cyclically converted via enzymatic pathways by epimerization, isomerization, and oxidization-reduction reactions. ${ }^{9-12}$ Notably, ketose 3-epimerase plays an irreplaceable role in the bioconversion of rare sugars, in which both D-tagatose 3-epimerase (DTE, EC 5.1.3.-) and D-allulose 3-epimerase (DAE, also named as DPE, EC 5.3.1) family enzymes can catalyze the reversible interconversion of D-allulose and D-sorbose with D-fructose and D-tagatose, respectively. Furthermore, the L-ribulose 3-epimerases (LREs) from M. loti and T. maritima, which showed the substrate specificity for L-ribulose, were also reported to convert D-fructose to D-allulose. ${ }^{\mathbf{1 3}, 14}$ DTE/DAE family enzymes require ions $\left(\mathrm{Co}^{2+}\right.$ or $\left.\mathrm{Mn}^{2+}\right)$ for their catalytic activity. ${ }^{15,16}$ Genes encoding DTE/DAE family enzymes have been identified in $P$. cichorii, ${ }^{17}$ R. sphaeroides,${ }^{18}$ A. tumefaciens, ${ }^{15}$ A. globiformis,${ }^{19}$ Agrobacterium sp. ${ }^{20}$ C. bolteae,${ }^{21}$ C. cellulolyticum,${ }^{16}$ C. scindens,${ }^{22}$ Clostridium sp. ${ }^{23}$ Desmospora sp. ${ }^{24}$ F. plautii, ${ }^{25}$ Ruminococcus sp. ${ }^{26}$ and $T$. primitia $^{27}$ However, only several crystal structures have been solved, including $C$. cellulolyticum $\mathrm{DAE},{ }^{28} A$. tumefaciens $\mathrm{DAE},{ }^{29}$ P. cichorii DTE, ${ }^{30}$ M. loti $\mathrm{LRE}^{31}$ and T. maritima LRE. ${ }^{14}$ The catalytic mechanism was also proposed based on the structural information. A C3-O3 proton-exchange mechanism 
was proposed, in which one Glu removes a proton from C-3 to generate a cis-enediolate intermediate via $\mathrm{O}-3$, and then another Glu protonates C-3 from the opposite side. ${ }^{29}$

Compared with the enzymatic synthesis of rare sugars, bioconversion using whole cells eliminates the purification steps, and it is considered to be more commercially competitive. Furthermore, whole recombinant cells of Escherichia coli expressing DTE/DAE from C. bolteae,${ }^{21}$ C. cellulolyticum, ${ }^{\mathbf{1 6}}$ and $A$. tumefaciens $^{32}$ were investigated, showing that whole cells have showed greater stability and resistance to environmental perturbations than the isolated enzymes. A novel putative Dtagatose 3-epimerase gene from Sinorhizobium sp. (sDTE, 882 bp, 293 amino acids) has been identified and is $32.1 \mathrm{kDa}$ without a signal peptide. To understand the relationship between the 3-D structure and biochemical characterization of
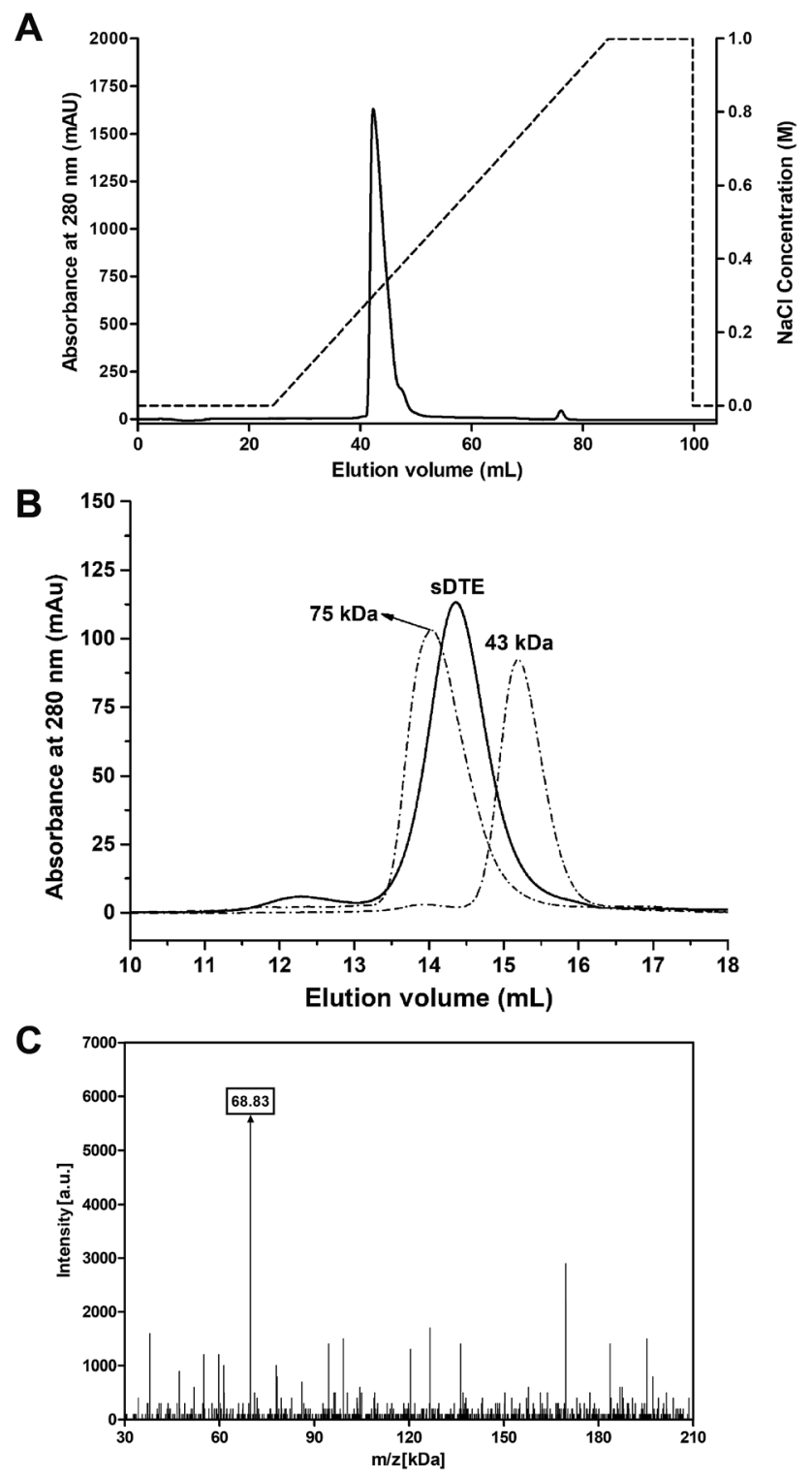

Fig. 1 Purification of sDTE by anion-exchange (A) and size-exclusion chromatography (B). Protein markers of ovalbumin $(43.0 \mathrm{kDa})$ and conalbumin $(75.0 \mathrm{kDa}$ ) were used. (C) MALDI-TOF spectra of sDTE.
sDTE, we analyzed its structural model and substrate specificity. The biocatalytic production of D-allulose using resting cells was also investigated.

\section{Materials and methods}

\section{Cloning and expression}

The sDTE gene (GenBank ID: NZ_CP016452.1; Protein ID: WP_069063284.1) was synthesized by Genewiz (Suzhou, China), and was inserted into the vector pET28a(+) (Novagen, Madison, WI, USA) with NdeI and EcoRI sites. The E. coli BL21 (DE3) cells harboring the sDTE gene were grown on Luria-Bertani (LB) medium supplemented $50 \mathrm{mg} \mathrm{L}{ }^{-1}$ kanamycin at $37{ }^{\circ} \mathrm{C}$ until the $\mathrm{OD}_{600}$ value reached 0.6 to 0.8 and then the recombinant cells were induced at $16{ }^{\circ} \mathrm{C}$ for $20 \mathrm{~h}$ by an addition isopropyl $\beta$-D-1thiogalactopyranoside (IPTG) to a final concentration of $0.5 \mathrm{mM}$. The cells were then collected from culture broth by centrifugation at $4{ }^{\circ} \mathrm{C}$ for $10 \mathrm{~min}$ at $5000 \times g$ and washed twice using lysis buffer (20 mM Tris- $\mathrm{HCl}$ pH 8.0) containing $10 \mathrm{mM}$ imidazole, $500 \mathrm{mM} \mathrm{NaCl}$, and $1 \mathrm{mM}$ dithiothreitol (DTT).

\section{Purification of the SDTE enzyme}

The recombinant $E$. coli cells were re-suspended in lysis buffer, and then disrupted by sonication for $10 \mathrm{~min}$ (pulse on $2.5 \mathrm{~s}$ and pulse off $3.0 \mathrm{~s}$ ). Cell debris was cleared by centrifugation (40 000 $\times g, 30 \mathrm{~min}, 4{ }^{\circ} \mathrm{C}$ ) and passage through a $0.45 \mu \mathrm{m}$ filters. The supernatants were loaded onto a column containing Ni-NTA Superflow resin (Qiagen, Hilden, Germany) equilibrated with lysis buffer. After the unbound proteins were washed with $10 \mathrm{~mL}$ lysis buffer, the recombinant enzyme was eluted from the column with $15 \mathrm{~mL}$ elution buffer (20 mM Tris-HCl pH 8.0) containing $300 \mathrm{mM}$ imidazole, $100 \mathrm{mM} \mathrm{NaCl}$, and $1 \mathrm{mM}$ DTT. The eluted solution was dialyzed overnight against $20 \mathrm{mM}$ Tris$\mathrm{HCl} \mathrm{pH} 8.0,1 \mathrm{mM}$ DTT to remove imidazole and was further purified by ion exchange using Resource Q (volume: $6 \mathrm{~mL}$, flow rate: $5 \mathrm{~mL} \mathrm{~min}^{-1}$, GE Healthcare). The molecular mass was estimated by gel filtration chromatography on a Superdex 200 Increase column 10/300 GL (volume: $24 \mathrm{~mL}$, GE Healthcare) and equilibrated with buffer ( $20 \mathrm{mM}$ Tris- $\mathrm{HCl}$ pH 8.0, $100 \mathrm{mM} \mathrm{NaCl}$, and $1 \mathrm{mM}$ DTT) at a flow rate of $0.5 \mathrm{~mL} \min ^{-1} \cdot{ }^{33,34}$ The total protein concentration was determined using a BCA assay kit (Solarbio, China) and bovine serum albumin was used as the standard. The amount and purity of the target protein were determined by sodium dodecyl sulfate polyacrylamide gel electrophoresis (SDS-PAGE) and Coomassie Brilliant Blue staining. The purified enzyme was used for activity assays.

\section{MALDI-TOF mass spectrometry}

A total of $1 \mu \mathrm{L}$ of enzyme sample solution was spotted onto a MALDI target containing $1 \mu \mathrm{L}$ saturated alpha-cyano-4hydroxycinnamic acid (HCCA) in a solvent mixture comprising acetonitrile and water $(70: 30, \mathrm{v} / \mathrm{v})$ with $0.01 \%$ trifluoroacetic acid. The sample was analyzed using an ultrafleXtreme TOF/ TOF instrument (Bruker Daltonics, Germany) operating in linear mode. MALDI-TOF spectra were analyzed using FlexAnalysis software (Bruker Daltonics). 


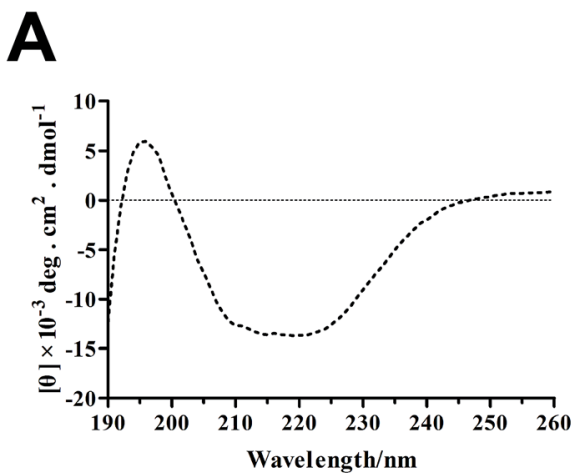

B

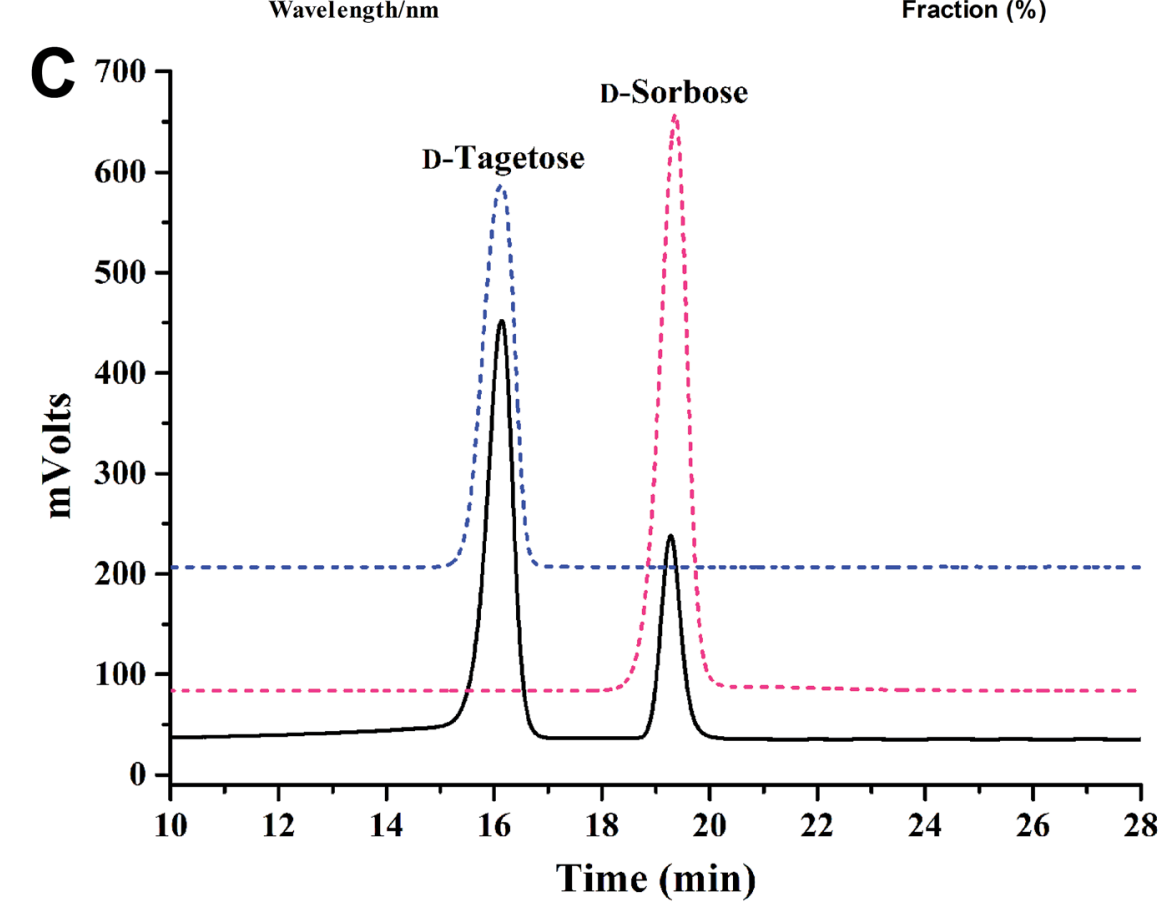

Fig. 2 CD spectra (A) and secondary structure assignments (B) of SDTE; (C) confirmation of the enzymatic conversion of D-tagatose using HPLC.

\section{Site-directed mutagenesis}

The mutant SDTE were constructed by one-step PCR reactions using a pair of complementary primers (Table S1 $\dagger$ ). The amplified PCR products were treated with DpnI to digest the template plasmid, and the DpnI-treated mixture is then used to transform into $E$. coli JM109 cells to yield plasmids encoding genes. The mutation constructs were verified by DNA sequencing (Genewiz, China). The plasmids containing mutant genes were introduced into $E$. coli BL21 (DE3) for protein expression and the mutant proteins were purified by the method for wild-type enzyme described above.

\section{CD spectroscopy}

The circular dichroism (CD) spectra (MOS-450, Biologic, Claix, Charente, France) were recorded in the far-UV (190-260 nm) with a $1 \mathrm{~mm}$ path-length cell at $25^{\circ} \mathrm{C}$. Four spectra of the far-UV $\mathrm{CD}$ of were recorded at a scan rate of $1 \mathrm{~nm} \mathrm{~s}^{-1}$ with a response of $1 \mathrm{~s}$ and bandwidth of $0.1 \mathrm{~nm}$ in $20 \mathrm{mM}$ Tris- $\mathrm{HCl}$ ( $\mathrm{pH} \mathrm{8.0)}$, $100 \mathrm{mM} \mathrm{NaCl}$, and $1 \mathrm{mM}$ DTT containing sDTE $\left(0.10 \mathrm{mg} \mathrm{mL}^{-1}\right)$. The reported spectra were the averages of four scans that were

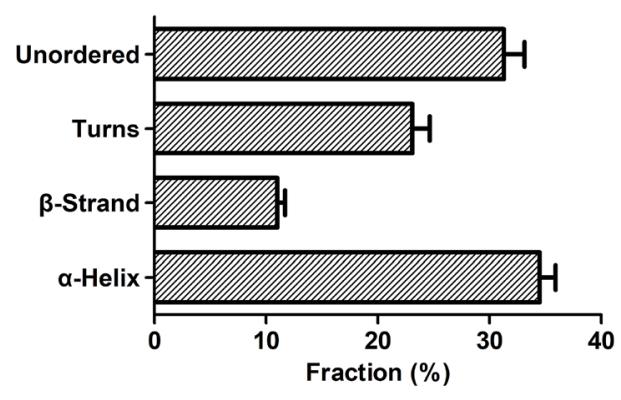

Sorbose 
A

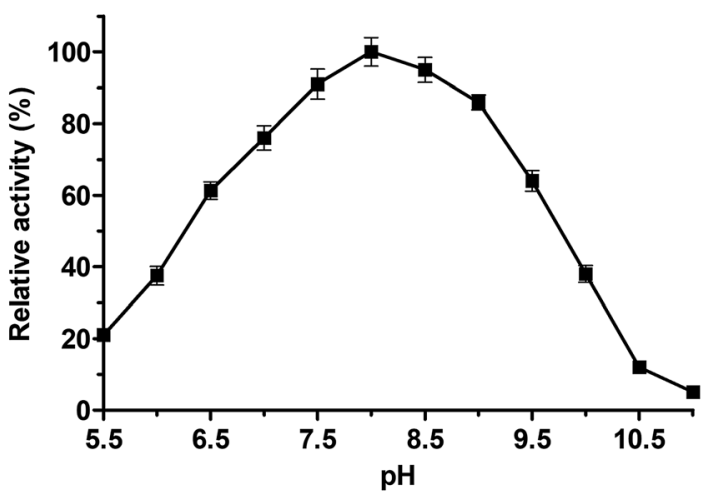

B

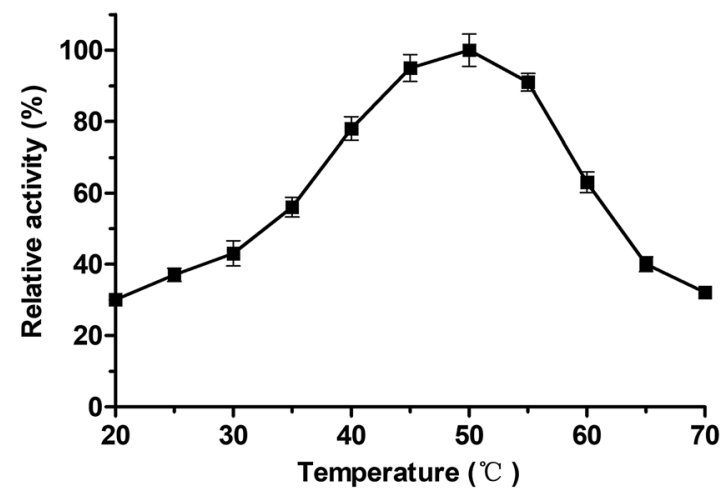

$\mathbf{C}$

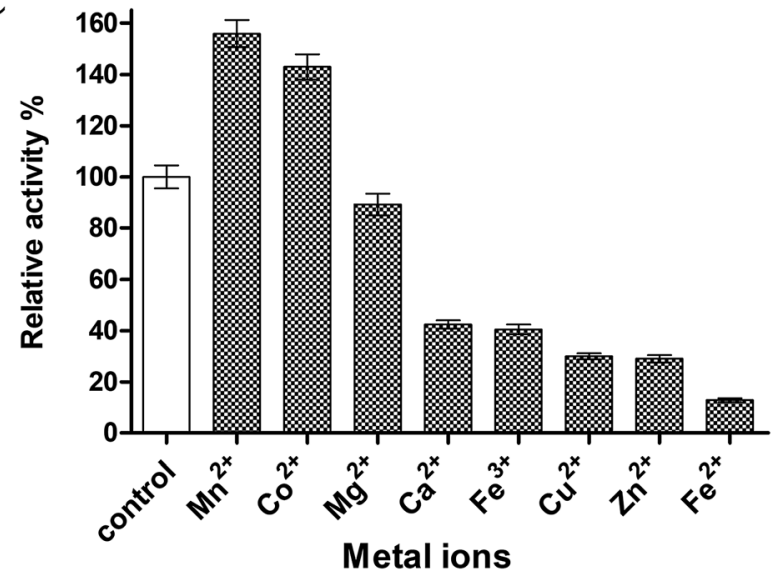

Fig. 3 Effect of $\mathrm{pH}(\mathrm{A})$, temperature (B) and metal ions (C) on activity of sDTE. The activity of purified SDTE using AKTA system was determined in standard assay conditions as control. All assays were repeated three times, and the data are shown as mean \pm S.D.

reaction mixtures were incubated in a boiling water bath for $5 \mathrm{~min}$ and then D-sorbose was measured using HPLC.

To investigate the optimal temperature, the activity was determined by incubating sDTE at 20 to $70{ }^{\circ} \mathrm{C}$. The optimal $\mathrm{pH}$ of the purified enzyme was determined at $50{ }^{\circ} \mathrm{C}$ for $10 \mathrm{~min}$ across a $\mathrm{pH}$ range of 5.5 to 11.0, using four buffers systems (MES: pH 5.5-6.5, PBS: 7.0-8.0, Tris-HCl: 8.5-9.0, CAPS: pH 9.511.0). To determine the effect of various metal ions on the activity of SDTE, the enzyme solution purified was treated with EDTA for $8 \mathrm{~h}$ and dialyzed against $20 \mathrm{mM}$ Tris- $\mathrm{HCl}$ buffer $(\mathrm{pH}$ 8.0). The reaction system contains the following ions at $1 \mathrm{mM}$ : $\mathrm{Ca}^{2+}, \mathrm{Mg}^{2+}, \mathrm{Mn}^{2+}, \mathrm{Co}^{2+}, \mathrm{Zn}^{2+}, \mathrm{Cu}^{2+}, \mathrm{Fe}^{2+}$, and $\mathrm{Fe}^{3+}$, respectively. The conversion of the wild-type enzyme in $20 \mathrm{mM}$ Tris- $\mathrm{HCl}$ buffer at $50{ }^{\circ} \mathrm{C}$ without adding metals was defined as $100 \%$. The activity was then measured as described above. All assays were repeated three separate times, and the data are shown as the means \pm S.D.

\section{Structural homologous model of SDTE}

The homology homologous model of SDTE was generated using Modeller 9.9. ${ }^{36}$ The crystal structure of Pseudomonas cichorii DTE (PcDTE, PDB ID: 4XSL, 1.6 ̊) was chosen as template, which has $29 \%$ sequence identity to the target protein SDTE. A sequence alignment was automatically generated between PcDTE and sDTE using align2d command. Subsequently, homology modeling was performed using the automodel command. Thereafter, each model was optimized and simulated annealing MD simulations were used to refine the structure. Finally, the best model was chosen from using the values of the Modeller objective function and the DOPE assessment scores. The generated model structures were visualized by utilizing the PyMoL software (http://www.pymol.org). ${ }^{37}$

\section{Bioconversion of $\mathrm{D}$-fructose into $\mathrm{D}$-allulose using isolated SDTE enzyme and resting cells}

Enzymatic synthesis of $\mathrm{D}$-allulose was carried out in a $10 \mathrm{~mL}$ reaction system containing $5 \mu \mathrm{M}$ purified sDTE, and $750 \mathrm{~g} \mathrm{~L}^{-1} \mathrm{D}^{-}$ fructose at optimal conditions. The progress of the enzymatic conversion was determined every $10 \mathrm{~min}$ for $60 \mathrm{~min}$. For the high production of D-allulose from D-fructose, recombinant $E$. coli BL21 (DE3) whole cells harboring sDTE were cultivated as described above, harvested, and washed twice with $20 \mathrm{mM}$ Tris$\mathrm{HCl}$ ( $\mathrm{pH}$ 8.0). The biotransformation was conducted in $20 \mathrm{mM}$ Tris-HCl (pH 8.0) containing $750 \mathrm{~g} \mathrm{~L}^{-1}$ D-fructose and $30 \mathrm{mg}$ of cells (wet weight per $\mathrm{mL}$ ). Samples comprising $1.0 \mathrm{~mL}$ of the resting cell suspension were drawn every $10 \mathrm{~min}$ for $60 \mathrm{~min}$. A fed-batch strategy was used to investigate the product yield with an initial $\mathrm{D}$-fructose concentration of $750 \mathrm{~g} \mathrm{~L}^{-1}$. Sampling was drawn every $20 \mathrm{~min}$ for $180 \mathrm{~min}$. Cells were disrupted by sonication, and were centrifuged at $40000 \times g$ and $4{ }^{\circ} \mathrm{C}$ for $30 \mathrm{~min}$ to remove the cell debris. The samples were diluted for HPLC analysis. All experiments were carried out in triplicates using $E$. coli BL21 (DE3) cells without the sDTE gene as controls.

\section{Results and discussion}

\section{Characterization of SDTE}

The recombinant SDTE was successfully overexpressed in E. coli BL21 (DE3), and was purified using Ni-NTA Superflow resin, followed by anion-exchange chromatography on a Resource Q column (Fig. 1A). The molecular mass of sDTE was analyzed by a size exclusion column, and a single peak corresponding to a dimer was observed (Fig. 1B). The molecular mass was further estimated by MALDI-TOF mass spectrometry, revealing a mass 
D-tagatose

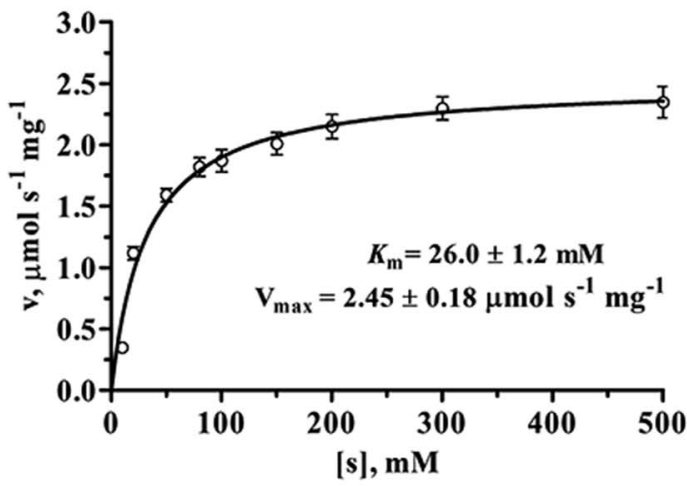

D-allulose

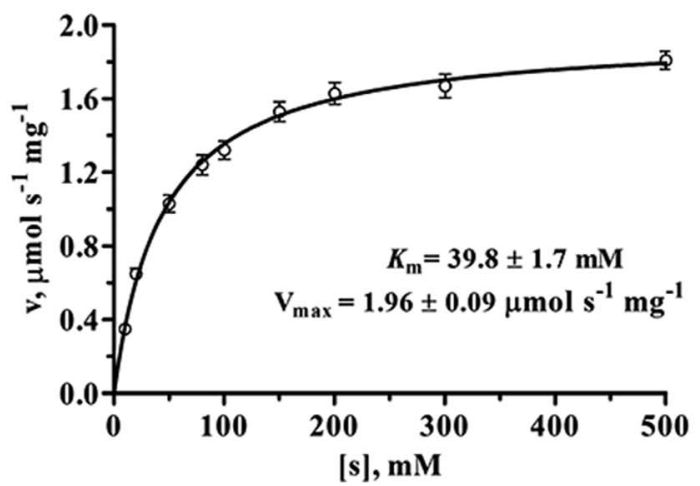

D-sorbose

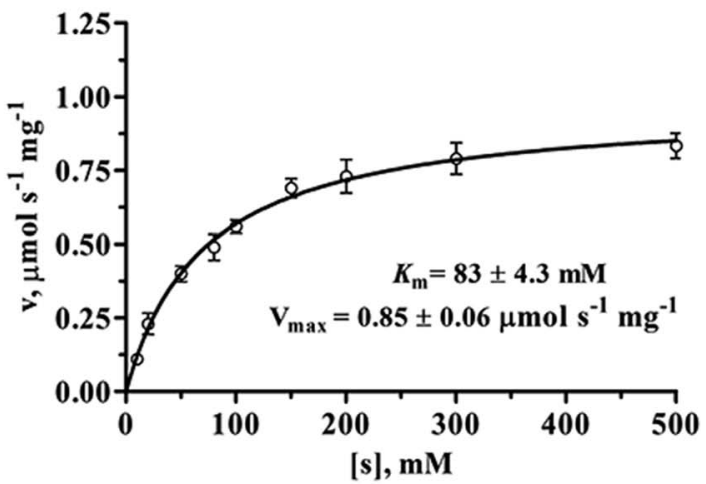

D-fructose

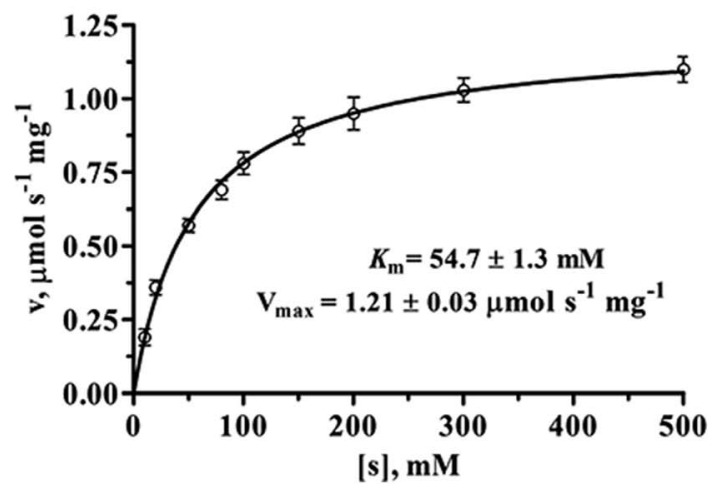

Fig. 4 Michaelis-Menten plots of sDTE toward D-tagatose, D-sorbose, D-allulose and D-fructose. All assays were repeated three times, and the data are shown as mean \pm S.D.

of approximately $68.83 \mathrm{kDa}$ (Fig. 1C). This value was about two times the molecular mass of recombinant SDTE $(34.44 \mathrm{kDa})$, including a tag of 21 residues (MGSSHHHHHHHSSGLVPRGSH) at the $\mathrm{N}$-terminus. Therefore, the recombinant enzyme is a dimer, which is the same as the DTEase from P. cichorii. ${ }^{17}$

The secondary structural features of purified sDTE were shown in Fig. 2A and B using CD spectroscopy, which showed a positive peak around $198 \mathrm{~nm}$. The secondary structural content of the SDTE was $35.1 \% \alpha$-helix, $11 \% \beta$-sheet, and $31.3 \%$ unstructured regions, respectively. The reaction product was analyzed by HPLC, which revealed a single peak of D-sorbose, with a retention time of 19.26 min (Fig. 2C).

The optimum $\mathrm{pH}$ and temperature for SDTE are shown in Fig. 3. Recombinant enzyme was optimally active in Tris- $\mathrm{HCl}$ buffer $\mathrm{pH} 8.0$ and showed activity at $\mathrm{pH}$ values from 5.5 to 11.0, indicating that the sDTE had an extensive $\mathrm{pH}$ range (Fig. 3A).
The sDTE showed a maximum activity at $50{ }^{\circ} \mathrm{C}$, and the activity significantly decreased at temperatures over $60^{\circ} \mathrm{C}$ (Fig. 3B). The effects of various metal ions on the activity of sDTE are displayed in Fig. 3C. The activity of sDTE increased to $156 \%$ and $143 \%$ in the presence of $1 \mathrm{mM} \mathrm{MnCl}_{2}$ and $\mathrm{CoCl}_{2}$, respectively, suggesting that $\mathrm{Mn}^{2+}$ and $\mathrm{Co}^{2+}$ might participate in the catalytic reaction. The activity of SDTE was unchanged in the presence of $\mathrm{MgCl}_{2}$, but it decreased when $\mathrm{CaCl}_{2}, \mathrm{FeCl}_{3}, \mathrm{CuCl}_{2}, \mathrm{ZnCl}_{2}$, and $\mathrm{FeCl}_{2}$ were added to the reaction system. The results thus support the previously published view that DTE family members are metalloenzymes. ${ }^{15,16}$

The kinetic parameters of SDTE with four substrates were determined by non-linear regression fitting of MichaelisMenten equation with GraphPad Prism 5.0 software (GraphPad Software, San Diego, CA). As shown in Fig. 4 and Table 1, sDTE was characterized by $K_{\mathrm{m}}$ values of between 26 and $83 \mathrm{mM}$, and

Table 1 Kinetic parameters of SDTE wild-type on four substrates ${ }^{a}$

\begin{tabular}{|c|c|c|c|c|}
\hline Substrates & $K_{\mathrm{m}}(\mathrm{mM})$ & $k_{\text {cat }}\left(\mathrm{s}^{-1}\right)$ & $k_{\text {cat }} / K_{\mathrm{m}}\left(\mathrm{s}^{-1} \mathrm{mM}^{-1}\right)$ & Relative activity (\%) \\
\hline D-Fructose & $54.7 \pm 1.3$ & $50 \pm 0.82$ & 0.91 & $46 \pm 0.96$ \\
\hline D-Allulose & $39.8 \pm 1.7$ & $78.4 \pm 2.3$ & 1.97 & $75 \pm 2.2$ \\
\hline D-Tagatose & $26.0 \pm 1.2$ & $98.3 \pm 0.92$ & 3.78 & $100 \pm 3.1$ \\
\hline D-Sorbose & $83 \pm 4.3$ & $34.0 \pm 0.92$ & 0.41 & $19 \pm 0.6$ \\
\hline
\end{tabular}

${ }^{a}$ All assays were repeated three times, and the data are shown as mean \pm S.D. 
Table 2 Comparison of some enzyme properties between sDTE and other DTEs or DAEs from different sources ${ }^{f}$

\begin{tabular}{|c|c|c|c|c|c|c|}
\hline \multicolumn{2}{|c|}{ Strains for enzyme source } & A. tumefaciens ${ }^{15}$ & R. sphaeroides $^{18}$ & Ruminococcus sp. ${ }^{26}$ & Agrobacterium sp. ${ }^{20}$ & Sinorhizobium sp. ${ }^{a}$ \\
\hline \multicolumn{2}{|l|}{ Optimum pH } & 8.0 & 8.5 & $7.5-8.0$ & $7.5-8.0$ & 8.0 \\
\hline \multicolumn{2}{|c|}{ Optimum temperature $\left({ }^{\circ} \mathrm{C}\right)$} & 50 & 35 & 60 & $55-60$ & 50 \\
\hline \multicolumn{2}{|c|}{ Most suitable substrate } & D-Allulose & D-Fructose & D-Allulose & D-Allulose & D-Tagatose \\
\hline \multicolumn{2}{|c|}{ MW of native protein } & 132 (tetramer) & 128 (tetramer) & 128 (tetramer) & 132 (tetramer) & 68 (dimer) \\
\hline$k_{\text {cat }}\left(\mathrm{s}^{-1}\right)$ & D-Allulose & 39.7 & 20.5 & 40.5 & NR & 78.4 \\
\hline & D-Fructose & 24 & 78 & 216 & 110 & 54.7 \\
\hline \multirow[t]{2}{*}{$k_{\mathrm{ca}} / K_{\mathrm{m}}\left(\mathrm{mM}^{-1} \mathrm{~s}^{-1}\right)$} & D-Allulose & 3.3 & 0.1 & 0.84 & NR & 1.97 \\
\hline & D-Fructose & 1.44 & 0.55 & 0.28 & 0.98 & 0.91 \\
\hline \multicolumn{2}{|c|}{ Productivity of D-allulose $\left(\mathrm{g} \mathrm{L}^{-1}\right)$} & $230^{b}$ & $118^{b}$ & $125^{c}$ & $150^{c}$ & $\begin{array}{l}260^{d} \\
476^{e}\end{array}$ \\
\hline
\end{tabular}

${ }^{a}$ This study. ${ }^{b}$ Produced from D-fructose with initial concentration of $700 \mathrm{~g} \mathrm{~L}^{-1}$. ${ }^{c}$ Produced from $\mathrm{D}$-fructose with initial concentration of $500 \mathrm{~g} \mathrm{~L}^{-1}$. ${ }^{d}$ Produced from D-fructose with initial concentration of $750 \mathrm{~g} \mathrm{~L}^{-1} .{ }^{e}$ Produced from D-fructose using a fed-batch bioconversion. ${ }^{f}$ NR, not reported.

catalytic efficiencies $k_{\text {cat }} / K_{\mathrm{m}}$ of between 0.41 and $3.78 \mathrm{~s}^{-1} \mathrm{mM}^{-1}$. SDTE showed a high activity toward D-tagatose, with a Michaelis constant of $26 \mathrm{mM}$ and catalytic efficiency of $3.78 \mathrm{~s}^{-1} \mathrm{mM}^{-1}$, which was four times of that of D-fructose, with a Michaelis constant of $54.7 \mathrm{mM}$ and catalytic efficiency of $0.91 \mathrm{~s}^{-1} \mathrm{mM}^{-1}$. SDTE showed low catalytic activity toward D-sorbose, the value of $k_{\text {cat }} / K_{\mathrm{m}}$ was $0.41 \mathrm{~s}^{-1} \mathrm{mM}^{-1}$. For D-allulose, the $k_{\text {cat }}, K_{\mathrm{m}}$, and $k_{\text {cat }} / K_{\mathrm{m}}$ are $78.4 \mathrm{~s}^{-1}, 39.8 \mathrm{mM}$, and $1.97 \mathrm{~s}^{-1} \mathrm{mM}^{-1}$, respectively, which is familiar to that of other DTEases (Table 2). ${ }^{15,16,19,23}$ Enzymes can be affected by temperature and $\mathrm{pH}$ in a variety of ways, for example, complex effects on the chemistry of catalysis and also on the structure, folding and stability of the protein. ${ }^{38}$ However, we only determined $k_{\text {cat }}$ and $K_{\mathrm{m}}$ at optimum temperature and $\mathrm{pH}$. We compared the enzymatic properties of SDTE with family enzymes in Table 2 . sDTE shows optimal $\mathrm{pH}$ and temperature at 8.0 and $50{ }^{\circ} \mathrm{C}$, and requires metal ions $\left(\mathrm{Co}^{2+}\right.$ or $\mathrm{Mn}^{2+}$ ) for enzymatic activities. sDTE is a dimer (68 $\left.\mathrm{kDa}\right)$ in solution, which is different from other enzymes (tetramer). SDTE showed the substrate specificity toward D-tagatose with a higher productivity (476 $\mathrm{g} \mathrm{L}^{-1}$ D-allulose) in a fed-batch bioconversion than other enzymes.

\section{Homology model of SDTE}

Based on the crystal structure of $P$. cichorii D-tagatose 3-epimerase, which has $29 \%$ sequence identity to SDTE,$^{30}$ the structural homology model of SDTE was generated. We evaluated the 3D model of SDTE that the secondary structural content of the homology model was $42.8 \% \alpha$-helix and $9.4 \% \beta$-sheet, respectively (https://servicesn.mbi.ucla.edu/SAVES/). These data are a little different between CD spectroscopy and homology model because $\mathrm{pH}$, temperature, and the buffer may affect $\mathrm{CD}$ spectroscopy of SDTE. The structure of SDTE (Fig. 5A) constituted of eight $\beta$-strands, ten $\alpha$-helices, and one $3_{10}$ helix, in which eight characteristic units of $\beta$-strand/ $\alpha$-helix at the core of structural motif formed canonical TIM-barrel $(\beta / \alpha)_{8}$ fold with a cluster of $\beta$-strands surrounded by $\alpha$-helices. The additional two $\alpha$-helices of $\alpha 6$ and $\alpha 9$ and one $3_{10}$ helix were packed together along with the TIM barrel. The active site is located at the top of the TIM barrel.
The structural model structure of SDTE showed that four residues, Glu154, Asp187, Gln213, and Glu248, coordinated the $\mathrm{Mn}^{2+}$ metal ion (Fig. 5B). Glu154 and Gln213 were located in the $\beta 5$ and $\beta 7$ stands of the TIM-barrel, respectively. Asp187 and Glu248 were located in the loop region of active site. They form a hydrogen bond network of catalytic tetrad of SDTE for the hydride transfer reaction. These four residues are partly conserved in the DTE/DAE/LRE superfamily (Fig. S1 $\dagger$ ), and three of the residues Glu154, Asp187, and Glu248 were absolutely conserved. However, Gln215 was not conserved, and replaced by a histidine, in P. cichorii DTE, A. tumefaciens DAE, C. cellulolyticum DAE, and T. maritima LRE. ${ }^{\text {14-17 }}$ The carbonyl oxygen O-2 and hydroxyl group O-3 in the substrate D-fructose/D-tagatose coordinated $\mathrm{Mn}^{2+}$ in a bidentate manner (Fig. 5B). This structure model is consistent with the previously proposed mechanism of deprotonation/protonation by two Glu residues of Glu154 and Glu248. ${ }^{28-30}$ In briefly, The Glu248 residue coordinating the $\mathrm{Mn}^{2+}$ removes a proton from C3 to generate a cisenediolate intermediate, after which Glu154 protonates C3 from the opposite side.

\section{Substrate specificity of SDTE}

The hydroxyl group O-1 of the substrate D-tagatose/D-fructose formed hydrogen bonds with Arg219 and Glu160 (Fig. 6). The $\mathrm{Mn}^{2+}$ ion is coordinated by $\mathrm{O}-2$ and -3 of the substrate, possibly neutralizing the oxyanion $\mathrm{O}-2$ oxyanion a putative cis-enediolate intermediate. $\mathrm{O}-3$ is stabilized further by hydrogen bonds with the hydroxyl groups of Glu154 and Glu248. When modeling with D-fructose or D-tagatose, we found that D-fructose has the same configurations of $\mathrm{O}-1,-2$, and -3 as D-tagatose (Fig. 6A). Furthermore, the interactions between $\mathrm{D}$-fructose/D-tagatose at the $\mathrm{O}-1,-2$, and -3 positions and the enzyme were very similar to those observed in other DTE/DAE family enzymes. ${ }^{28-30}$ Therefore, SDTE strictly regulates the catalytic reaction at the positions 1, 2, and 3. However, the residues interacting with $\mathrm{D}^{-}$ tagatose/D-fructose at the hydroxyl groups of $0-4,-5$, and -6 positions were not conserved among the enzymes of this family. The substrate-enzyme interactions at $0-4,-5$, and -6 are essential for the substrate specificity. They control the spatial 

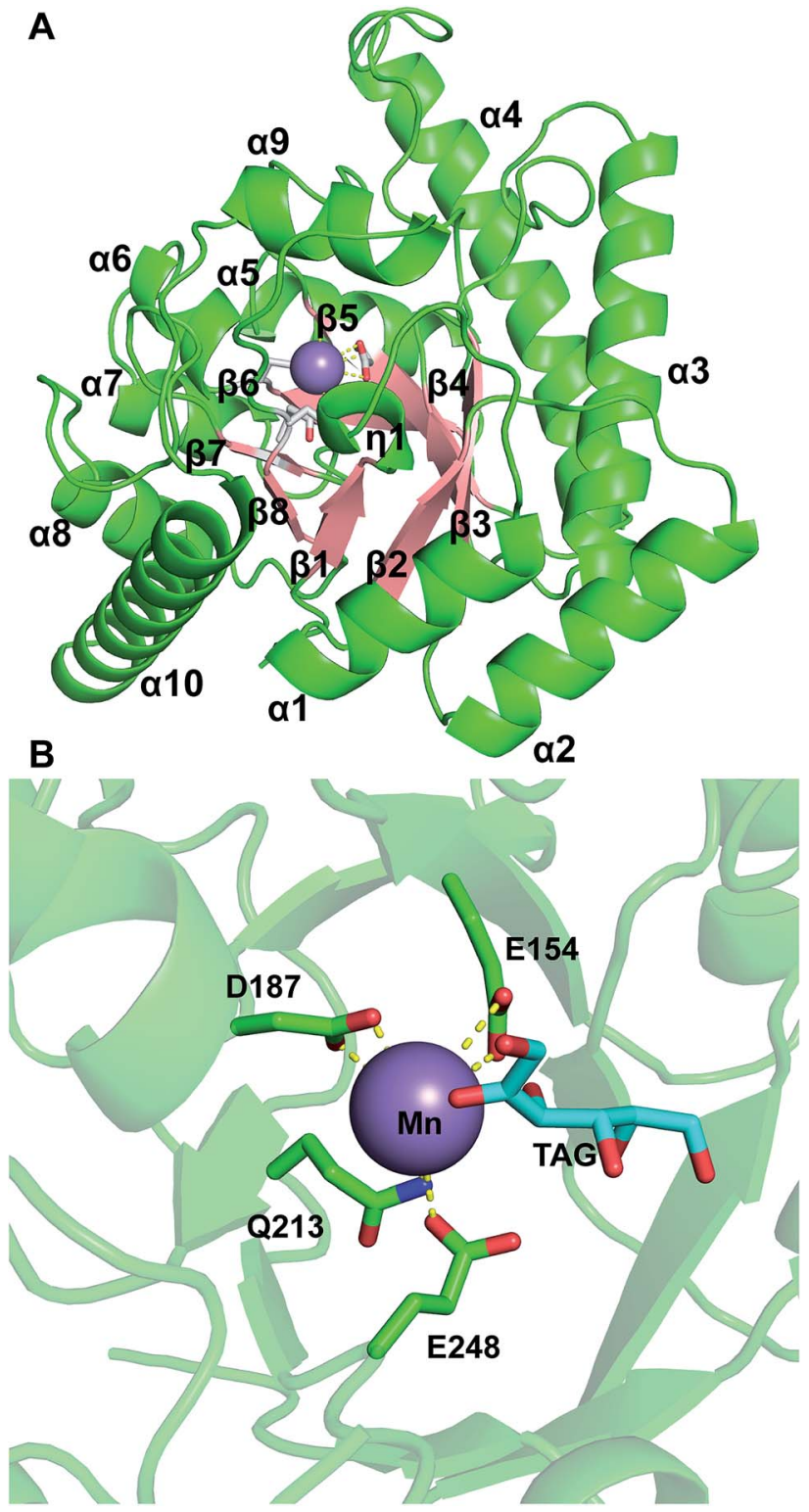

Fig. 5 Ribbon representation of the sDTE homology structure (A) and overview of the active site (B). The central $\beta$-strands and $\alpha$-helices of TIM barrel-fold are shown in salmon and green, respectively. Catalytic residues are shown as white and green sticks. The optimum substrate $\mathrm{D}$-tagatose is shown as cyan sticks. $\mathrm{Mn}(\|)$ is displayed as a purple sphere.

orientation and conformation of the various substrates. In particular, Arg65 formed a unique interaction with O-4 of Dfructose and $\mathrm{D}$-tagatose. Notably, $\mathrm{O}-4$ of $\mathrm{D}$-fructose formed two hydrogen bonds with NE and NH of Arg65. However, O-4 of Dtagatose was stabilized by Met9. Arg65 and Met9 were not conserved in the DTE/DAE family, and the mutation of R65G and M9A abolished the catalytic activity toward D-tagatose. The relative activities of R65G and M9A toward D-tagatose were 7.3\% and $5.1 \%$, respectively compared with wild-type sDTE (Fig. S2 $\dagger$ ). On the other hand, O-5 of D-tagatose interacted with Glu35 while $\mathrm{O}-5$ of D-fructose was anchored by the main chain of Gly104. The structural information supports the biochemical data, since SDTE showed high activity toward D-tagatose and D-
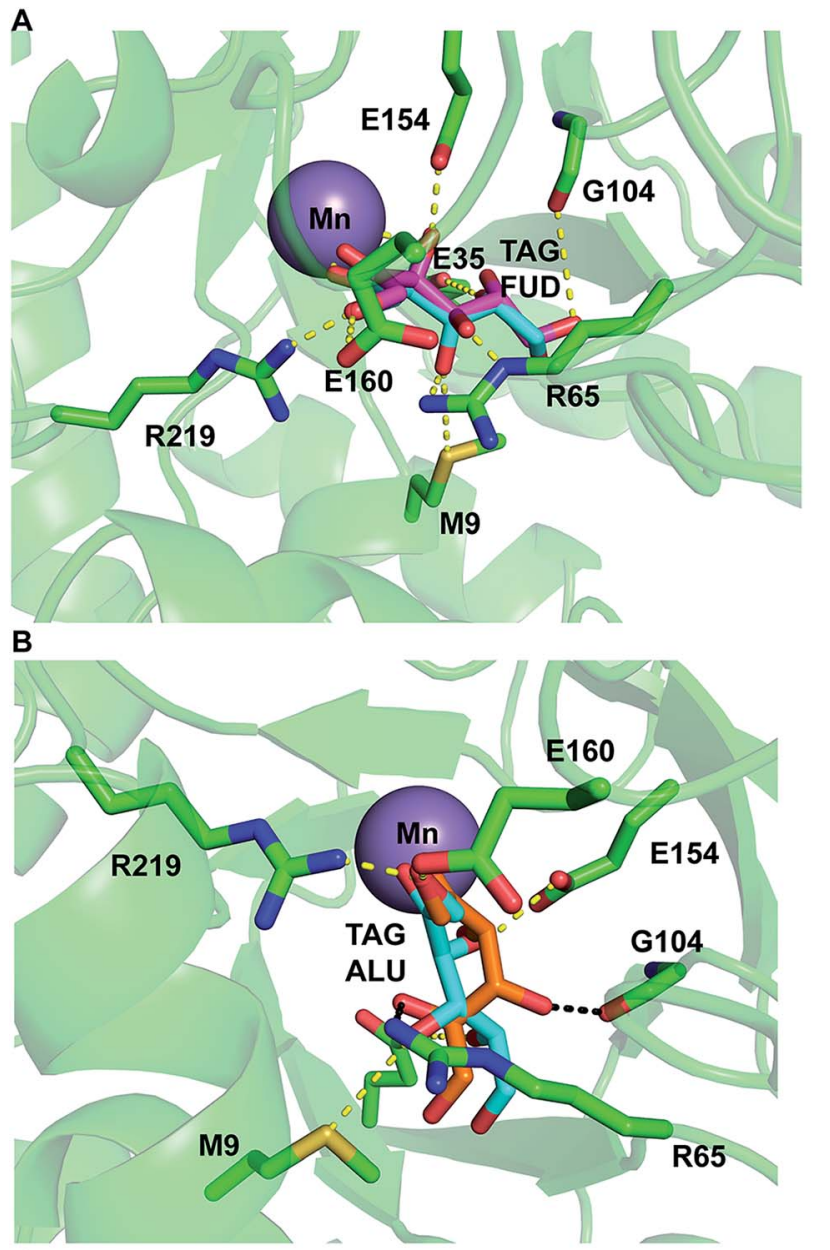

C

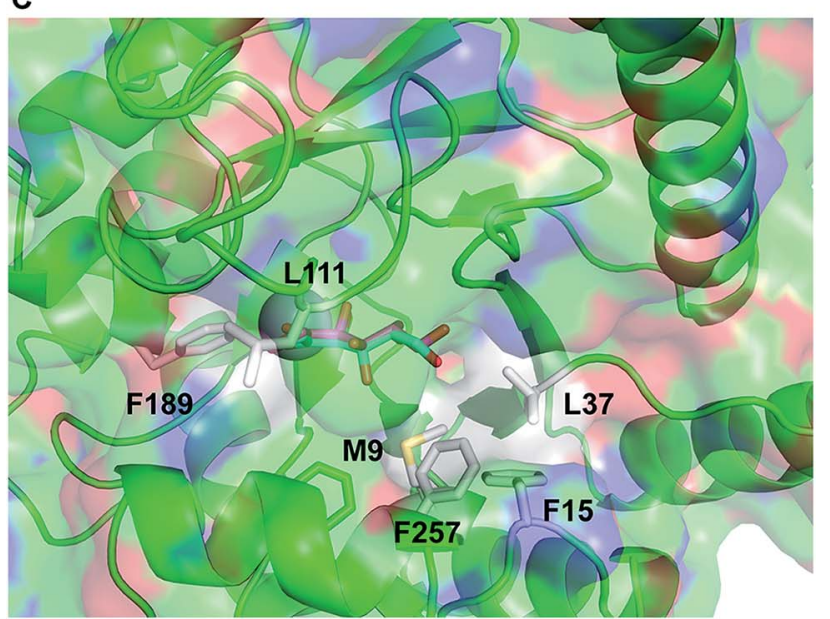

Fig. 6 Residues at substrate binding site of SDTE homology structure. (A) Residues of sDTE forms hydrogen bond with substrate. The substrates D-fructose and D-tagatose are shown as magenta and purple sticks, respectively. (B) The hydrogen bond interactions with sDTE and substrates. (C) The hydrophobic residues surrounding the substrate-binding site.

fructose. When comparing the superimposed of structural models of SDTE and C. cellulolyticum DAE, we found that Dallulose formed different hydrogen bond interactions with SDTE than D-tagatose (Fig. 6B), in which O-4 could interact with 
A

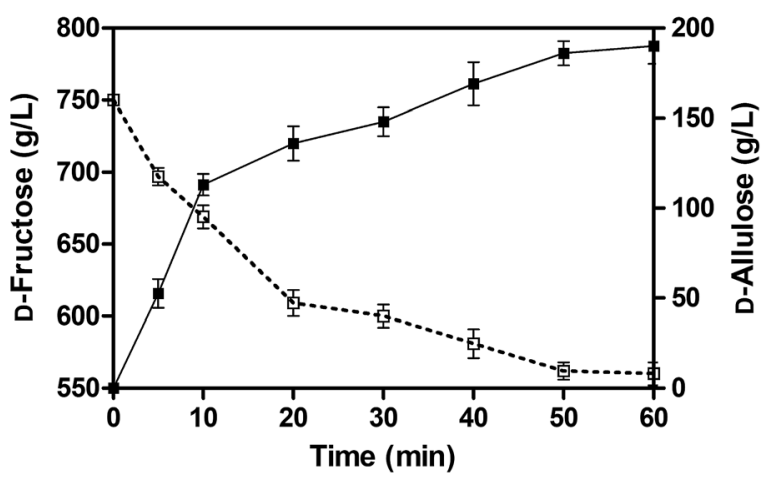

B

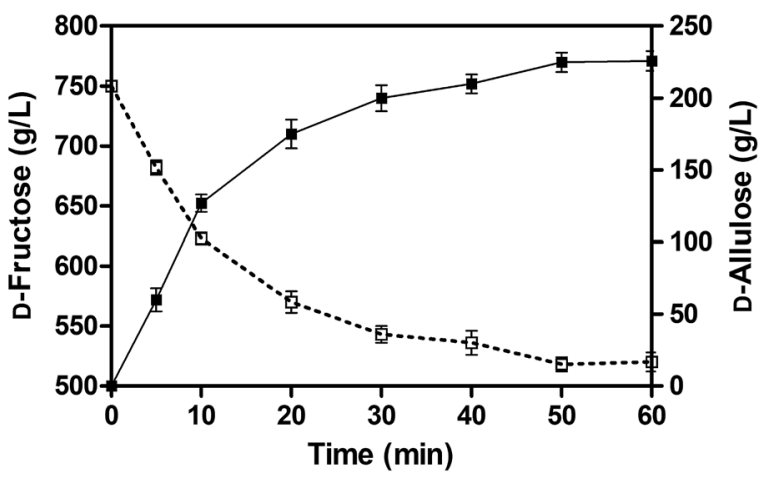

C

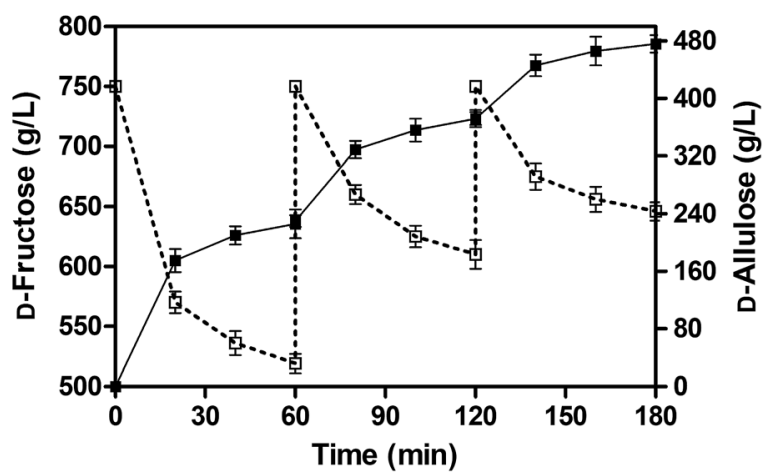

Fig. 7 Bioconversion of D-allulose by SDTE enzyme and resting cells (A) Enzymatic catalysis; (B) batch bioconversion; (C) fed-batch bioconversion. All assays were performed in triplicate with three independent measurements. Standard deviations of the biological replicates are represented by error bars.

Gly104 and O-5 was recognized by Arg65. There was no interaction between O-6 of D-allulose and SDTE. Some residues, such as Met9, Phe15, Leu37, Leu111, Phe189, and Phe257 formed a hydrophobic environment for the substrate recognition (Fig. 6C).

\section{Bioconversion of $\mathrm{D}$-fructose into $\mathrm{D}$-allulose using isolated SDTE enzyme and resting cells}

The biocatalytic synthesis of D-allulose using D-fructose as substrate was performed using isolated enzyme, as well as whole-cell batch, and fed-batch strategies (Fig. 7). Under enzymatic catalysis and batch bioconversion, the productivity of $\mathrm{D}^{-}$ allulose gradually increased and reached a plateau at $40 \mathrm{~min}$. The final productivity of $\mathrm{D}$-allulose reached 190 and $226 \mathrm{~g} \mathrm{~L}^{-1}$, respectively (Fig. 7A and B). The conversion rates were 25.3 and
$30.1 \%$, respectively. For high levers of product yield, the $\mathrm{D}^{-}$ allulose titers were improved using a fed-batch strategy (Fig. 7C). The reaction system was added with D-fructose every $60 \mathrm{~min}$ to continue the bioconversion. The yields of D-allulose showed a steady increase during the first two fed-batch processes, with productivity values of 223 and $372 \mathrm{~g} \mathrm{~L}^{-1}$ (bioconversion before $120 \mathrm{~min}$ ). The maximum $\mathrm{D}$-allulose concentrations $\left(476 \mathrm{~g} \mathrm{~L}^{-1}\right)$ was obtained at 180 min during the third fed-batch fermentation process (bioconversion from $120 \mathrm{~min}$ to $180 \mathrm{~min})$. The conversion rate reached $42.5 \%$. Therefore, fed-batch bioconversion with E. coli BL21 (DE3) expressing sDTE is an effective strategy to improve the D-allulose yield.

The bioconversion rate of D-allulose using A. tumefaciens DAE, at $32.9 \%$, compares favorably with other enzymes from this family, and was markedly higher than the $20 \%$ achieved with $P$. cichorii DTE. ${ }^{15,17}$ Yang et al. reported that turnover rate of D-allulose using A. tumefaciens DAE in $K$. marxianus was about $25.3 \% .^{39} R$. sphaeroides DTE shows the highest activity on $\mathrm{D}^{-}$ fructose, with a D-allulose bioconversion rate of $23 \%\left(40{ }^{\circ} \mathrm{C}, \mathrm{pH}\right.$ 9.0), which is also lower than that of $A$. tumefaciens DAE. ${ }^{40}$ In our research, sDTE showed a D-allulose bioconversion of 25.3 and $30.1 \%$ in pure-enzyme and whole-cell batch strategies, respectively. Furthermore, the bioconversion rate of sDTE-expressing cells reached $42.5 \%$ in fed-batch fermentation, which was higher than that of A. tumefaciens DAE.

\section{Conclusions}

The purified D-tagatose 3-epimerase from Sinorhizobium sp. catalyses mainly the epimerization of D-tagatose to D-sorbose at the C3 position. SDTE showed optimal activity in Tris- $\mathrm{HCl}$ buffer at $\mathrm{pH} 8.0$ and $50{ }^{\circ} \mathrm{C}$. The binding-site residues of SDTE interacting with the $\mathrm{O}-4,-5$, and -6 positions of the substrate are different, and Arg65 formed an interaction with O-4 of D-fructose and D-tagatose in a unique recognition mode. Furthermore, both the isolated sDTE enzyme and resting cells $E$. coli expressing sDTE showed high bioconversion rates in the production of D-allulose. Furthermore, the fed-batch strategy was an effective way to improve the D-allulose yield. The research on SDTE provides a valuable tool for commercial-scale manufacture of rare sugars in the future.

\section{Author contributions}

T. M., F. L., and H.-M. Q. designed the research; Z. Z., C. L., and X. L. performed the experiments; Z. Z., D. G., and X. W. analyzed data; H.-M. Q., F. L., and M. T. wrote the paper. All authors have approved the final version of the manuscript.

\section{Funding}

This work was supported by National Key Research and Development Project of China (2017YF0400304), National Natural Science Foundation of China (31771911), the Innovative Research Team of Tianjin Municipal Education Commission (TD13-5015), Natural Science Foundation of Tianjin 
(16JCQNJC09200) and the Overseas High-level Talents Program of Tianjin University of Science and Technology to H.-M. Qin, China.

\section{Ethics approval and consent to participate}

No animals or human subjects were used in the above research.

\section{Consent for publication}

Our manuscript does not contain any individual data in any form.

\section{Conflicts of interest}

The authors declare no competing financial interests.

\section{References}

1 W. Zhang, S. Yu, T. Zhang, B. Jiang and W. Mu, Trends Food Sci. Technol., 2016, 54, 127-137.

2 A. Hossain, F. Yamaguchi, T. Matsuo, I. Tsukamoto, Y. Toyoda, M. Ogawa, Y. Nagata and M. Tokuda, Pharmacol. Ther., 2015, 155, 49-59.

3 T. Matsuo, T. Tanaka, M. Hashiguchi, K. Izumori and H. Suzuki, Asia Pac. J. Clin. Nutr., 2003, 12, 225-231.

4 R. L. Whistler, P. P. Singh and W. C. Lake, Carbohydr. Res., 1974, 34, 200-202.

5 S. O'Charoen, S. Hayakawa and M. Ogawa, Int. J. Food Sci. Technol., 2015, 50, 194-202.

6 D.-K. Oh, Appl. Microbiol. Biotechnol., 2007, 76, 1-8.

7 Y. Lu, G. V. Levin and T. W. Donner, Diabetes, Obes. Metab., 2008, 10, 109-134.

8 M. Emmadi and S. S. Kulkarni, Nat. Prod. Rep., 2014, 31, 870879.

9 T. B. Granström, G. Takata, M. Tokuda and K. Izumori, J. Biosci. Bioeng., 2004, 97, 89-94.

10 K. Izumori, Naturwissenschaften, 2002, 89, 120-124.

11 K. Izumori, J. Biotechnol., 2006, 124, 717-722.

12 W. Mu, W. Zhang, Y. Feng, B. Jiang and L. Zhou, Appl. Microbiol. Biotechnol., 2012, 94, 1461-1467.

13 K. Uechi, G. Takata, Y. Fukai, A. Yoshihara and K. Morimoto, Biosci., Biotechnol., Biochem., 2013, 77, 511-515.

14 S.-M. Shin, T.-P. Cao, J.-M. Choi, S.-B. Kim, S.-J. Lee, S. H. Lee and D.-W. Lee, Appl. Environ. Microbiol., 2017, 83, e03291-16.

15 H. J. Kim, E. K. Hyun, Y. S. Kim, Y. J. Lee and D. K. Oh, Appl. Environ. Microbiol., 2006, 72, 981-985.

16 W. Mu, F. Chu, Q. Xing, S. Yu, L. Zhou and B. Jiang, J. Agric. Food Chem., 2011, 59, 7785-7792.

17 H. Itoh, H. Okaya, A. R. Khan, S. Tajima, S. Hayakawa and K. Izumori, Biosci., Biotechnol., Biochem., 1994, 58, 21682171.
18 Z. Qi, Z. Zhu, J.-W. Wang, S. Li, Q. Guo, P. Xu, F. Lu and H.-M. Qin, Microb. Cell Fact., 2017, 16, 193.

19 A. Yoshihara, T. Kozakai, T. Shintani, R. Matsutani, K. Ohtani, T. Iida, K. Akimitsu, K. Izumori and P. K. Gullapai, J. Biosci. Bioeng., 2017, 123, 170-176.

20 W.-C. Tseng, C.-N. Chen, C.-T. Hsu, H.-C. Lee, H.-Y. Fang, M.-J. Wang, Y.-H. $\mathrm{Wu}$ and T.-Y. Fang, Int. J. Biol. Macromol., 2018, 112, 767-774.

21 M. Jia, W. M. Mu, F. F. Chu, X. M. Zhang, B. Jiang, L. L. Zhou and T. Zhang, Appl. Microbiol. Biotechnol., 2014, 98, 717-725.

22 W. L. Zhang, D. Fang, Q. C. Xing, L. Zhou, B. Jiang and W. M. Mu, PLoS One, 2013, 8, e62987.

23 W. M. Mu, W. L. Zhang, D. Fang, L. Zhou, B. Jiang and T. Zhang, Biotechnol. Lett., 2013, 35, 1481-1486.

24 W. Zhang, D. Fang, T. Zhang, L. Zhou, B. Jiang and W. Mu, J. Agric. Food Chem., 2013, 61, 11468-11476.

25 C.-S. Park, T. Kim, S.-H. Hong, K.-C. Shin, K.-R. Kim and D.-K. Oh, PLoS One, 2016, 11, e0160044.

26 Y. Zhu, Y. Men, W. Bai, X. Li, L. Zhang, Y. Sun and Y. Ma, Biotechnol. Lett., 2012, 34, 1901-1906.

27 W. Zhang, T. Zhang, B. Jiang and W. Mu, J. Sci. Food Agric., 2016, 96, 49-56.

28 H.-C. Chan, Y. Zhu, Y. Hu, T.-P. Ko, C.-H. Huang, F. Ren, C.-C. Chen, Y. Ma, R.-T. Guo and Y. Sun, Protein Cell, 2012, 3, 123-131.

29 K. Kim, H.-J. Kim, D.-K. Oh, S.-S. Cha and S. Rhee, J. Mol. Biol., 2006, 361, 920-931.

30 H. Yoshida, M. Yamada, T. Nishitani, G. Takada, K. Izumori and S. Kamitori, J. Mol. Biol., 2007, 374, 443-453.

31 K. Uechi, H. Sakuraba, A. Yoshihara, K. Morimoto and G. Takata, Acta Crystallogr., Sect. D: Biol. Crystallogr., 2013, 69, 2330-2339.

32 C. S. Park, K. C. Shin and D. K. Oh, J. Biosci. Bioeng., 2016, 121, 186-190.

33 H.-M. Qin, T. Miyakawa, A. Inoue, R. Nishiyama, A. Nakamura, A. Asano, T. Ojima and M. Tanokura, Chem. Commun., 2018, 54, 555-558.

34 H.-M. Qin, T. Miyakawa, A. Inoue, R. Nishiyama, A. Nakamura, A. Asano, Y. Sawano, T. Ojima and M. Tanokura, J. Biol. Chem., 2017, 292, 2182-2190.

35 L. Whitmore and B. A. Wallace, Biopolymers, 2008, 89, 392400.

36 A. Šali and T. L. Blundell, J. Mol. Biol., 1993, 234, 779-815.

37 W. Humphrey, A. Dalke and K. Schulten, J. Mol. Graphics, 1996, 14, 33-38.

38 A. Cornish-Bowden, Fundamentals of Enzyme Kinetics, Portland Press, London, 1995.

39 P. Yang, X. Zhu, Z. Zheng, D. Mu, S. Jiang, S. Luo, Y. Wu and M. Du, World J. Microbiol. Biotechnol., 2018, 34, 65.

40 L. Zhang, W. Mu, B. Jiang and T. Zhang, Biotechnol. Lett., 2009, 31, 857-862. 